\title{
A New Conjugate Gradient Algorithm with Sufficient Descent Property for Unconstrained Optimization
}

\author{
XiaoPing Wu, ${ }^{1}$ LiYing Liu, ${ }^{2}$ FengJie Xie, ${ }^{1}$ and YongFei Li ${ }^{1}$ \\ ${ }^{1}$ School of Economic Management, Xian University of Posts and Telecommunications, Shaanxi, Xian 710061, China \\ ${ }^{2}$ School of Mathematics Science, Liaocheng University, Shandong, Liaocheng 252000, China \\ Correspondence should be addressed to XiaoPing Wu; wuxiaoping1978@126.com
}

Received 16 May 2015; Revised 24 September 2015; Accepted 29 September 2015

Academic Editor: Masoud Hajarian

Copyright (c) 2015 XiaoPing Wu et al. This is an open access article distributed under the Creative Commons Attribution License, which permits unrestricted use, distribution, and reproduction in any medium, provided the original work is properly cited.

A new nonlinear conjugate gradient formula, which satisfies the sufficient descent condition, for solving unconstrained optimization problem is proposed. The global convergence of the algorithm is established under weak Wolfe line search. Some numerical experiments show that this new WWPNPRP ${ }^{+}$algorithm is competitive to the SWPPRP ${ }^{+}$algorithm, the SWPHS algorithm, and the WWPDYHS ${ }^{+}$algorithm.

\section{Introduction}

In this paper, we consider the following unconstrained optimization problem:

$$
\min \left\{f(x) \mid x \in \mathfrak{R}^{n}\right\},
$$

where $f(x): \mathfrak{R}^{n} \rightarrow \mathfrak{R}$ is a twice continuously differentiable function whose gradient is denoted by $g(x): \mathfrak{R}^{n} \rightarrow \mathfrak{R}^{n}$. Its iterative formula is given by

$$
x_{k+1}=x_{k}+t_{k} d_{k}
$$

where

$$
d_{k}= \begin{cases}-g_{k} & \text { if } k=1, \\ -g_{k}+\beta_{k} d_{k-1} & \text { if } k \geq 2,\end{cases}
$$

and $t_{k}$ is a step size which is computed by carrying out a line search, $\beta_{k}$ is a scalar, and $g_{k}$ denotes $g\left(x_{k}\right)$. There are at least six famous formulas for $\beta_{k}$, which are given below:

$$
\begin{aligned}
& \beta_{k}^{\mathrm{HS}}=\frac{g_{k}^{T}\left(g_{k}-g_{k-1}\right)}{\left(g_{k}-g_{k-1}\right)^{T} d_{k-1}}, \\
& \beta_{k}^{\mathrm{FR}}=\frac{g_{k}^{T} g_{k}}{g_{k-1}^{T} g_{k-1}},
\end{aligned}
$$

$$
\begin{aligned}
& \beta_{k}^{\mathrm{PRP}}=\frac{g_{k}^{T}\left(g_{k}-g_{k-1}\right)}{g_{k-1}^{T} g_{k-1}}, \\
& \beta_{k}^{\mathrm{CD}}=-\frac{g_{k}^{T} g_{k}}{d_{k-1}^{T} g_{k-1}}, \\
& \beta_{k}^{\mathrm{LS}}=-\frac{g_{k}^{T}\left(g_{k}-g_{k-1}\right)}{d_{k-1}^{T} g_{k-1}}, \\
& \beta_{k}^{\mathrm{DY}}=\frac{g_{k}^{T} g_{k}}{\left(g_{k}-g_{k-1}\right)^{T} d_{k-1}} .
\end{aligned}
$$

To establish the global convergence results of the above conjugate gradient (CG) methods, it is usually required that the step size $t_{k}$ should satisfy some line search conditions, such as the weak Wolfe-Powell (WWP) line search

$$
\begin{gathered}
f\left(x_{k}+t_{k} d_{k}\right)-f\left(x_{k}\right) \leq \delta t_{k} g_{k}^{T} d_{k}, \\
g\left(x_{k}+t_{k} d_{k}\right)^{T} d_{k} \geq \sigma g_{k}^{T} d_{k},
\end{gathered}
$$

where $\delta \in(0,1 / 2)$ and $\sigma \in(\delta, 1)$, and strong Wolfe-Powell (SWP) line search (5) and

$$
\left|g\left(x_{k}+t_{k} d_{k}\right)^{T} d_{k}\right| \leq-\sigma g_{k}^{T} d_{k},
$$


where $\delta \in(0,1 / 2)$ and $\sigma \in(\delta, 1)$. Wolfe-Powell is referred to as Wolfe.

Considerable attentions have been made on the global convergence behaviors for the above methods. Zoutendijk [1] proved that the FR method with exact line search is globally convergent. Al-Baali [2] extended this result to the strong Wolfe line search conditions. In [3], Dai and Yuan proposed the DY method which produces a descent search direction at every iteration and converges globally provided that the line search satisfies the weak Wolfe conditions. In [4], Wei et al. discussed the global convergence of the PRP conjugate gradient method (CGM) with inexact line search for nonconvex unconstrained optimization. Recently, based on [5-7], Jiang et al. [8] proposed a hybrid CGM with

$$
\beta_{k}^{\mathrm{IHJ}}=\frac{\left\|g_{k}\right\|^{2}-\max \left\{0,\left(\left\|g_{k}\right\| /\left\|d_{k-1}\right\|\right) g_{k}^{T} d_{k-1},\left(\left\|g_{k}\right\| /\left\|g_{k-1}\right\|\right) g_{k}^{T} g_{k-1}\right\}}{d_{k-1}^{T}\left(g_{k}-g_{k-1}\right)} .
$$

Under the Wolfe line search, the method possesses global convergence and efficient numerical performance.

On some studies of the conjugate gradient methods, the sufficient descent condition

$$
g_{k}^{T} d_{k} \leq-c\left\|g_{k}\right\|^{2}, \quad c>0
$$

is often used to analyze the global convergence of the nonlinear conjugate gradient method with the inexact line search techniques. For instance, Touati-Ahmed and Storey [9], Al-Baali [2], Gilbert and Nocedal [10], and $\mathrm{Hu}$ and Storey [11] hinted that the sufficient descent condition may be crucial for conjugate gradient methods. Unfortunately, this condition is hard to hold. It has been showed that the PRP method with the strong Wolfe Powell line search does not ensure this condition at each iteration. So, Grippo and Lucidi [12] managed to find some line searches which ensure the sufficient descent condition, and they presented a new line search which ensures this condition. The convergence of the PRP method with this line search had been established. Yu et al. [13] analyzed the global convergence of modified PRP CGM with sufficient descent property. Gilbert and Nocedal [10] gave another way to discuss the global convergence of the PRP method with the weak Wolfe line search. By using a complicated line search, they were able to establish the global convergence result of the PRP and HS methods by restricting the parameter $\beta_{k}$ in (3), not allowed to be negative; that is,

$$
\beta_{k}^{+}=\max \left\{0, \beta_{k}^{\mathrm{PRP}}\right\},
$$

which yields a globally convergent CG method, being also computationally efficient [14]. In spite of the numerical efficiency of the PRP method, as an important defect, the method lacks the following descent property:

$$
g_{k}^{T} d_{k} \leq 0, \quad \forall k \geq 0,
$$

even for uniformly convex objective functions [15]. This motivated the researchers to pay much attention to finding some extensions of the PRP method with descent property. In this context, Yu et al. [16] proposed a modified form of $\beta_{k}^{\text {PRP }}$ as follows:

$$
\beta_{k}^{\mathrm{DPRP}}=\beta_{k}^{\mathrm{PRP}}-C \frac{\left\|y_{k}\right\|^{2}}{\left\|g_{k}\right\|^{4}} g_{k+1}^{T} d_{k}
$$

with a constant $C \geq 1 / 4$, leading to a CG method with the sufficient descent property. Dai and Kou [17] propose a family of conjugate gradient methods and an improved Wolfe line search; meanwhile, to accelerate the algorithm, an adaptive restart along negative gradients method is introduced. Jiang and Jian [18] proposed two modified CGMs with disturbance factors based on a variant of PRP method; the two proposed methods not only generate sufficient descent direction at each iteration but also converge globally for nonconvex minimization if the strong Wolfe line search is used. A new hybrid conjugate gradient method was presented for unconstrained optimization. The proposed method can generate decent directions at every iteration; moreover, this property is independent of the steplength line search. Under the Wolfe line search, the proposed method possesses global convergence [19].

The main purpose of this paper is to design an efficient algorithm which possesses the properties of global convergence, sufficient descent, and good numerical results. In next section, we present a new CG formula and give its properties. In Section 3, the new algorithm and its global convergence result will be established. To test and compare the numerical performance of the proposed method, in the last part of this work, a large amount of medium-scale numerical experiments are reported by tables and performance profiles.

\section{The Formula and Its Property}

Because sufficient descent condition (9) is a very nice and important property to analyze the global convergence of the CG methods, we hope to find $\beta_{k}$ such that $d_{k}$ satisfies (9). In the following, we propose a sequence $\left\{\beta_{k}\right\}$ and prove that it has such property. Firstly, we give a definition of a descent sequence (or a sufficient descent sequence): a sequence $\left\{\beta_{k}\right\}$ is called a descent sequence (or a sufficient descent sequence) for the CG methods if there exists a constant $\tau \in(0,1)$ (or $\tau \in[0,1))$ such that, for all $k \geq 2$,

$$
\beta_{k} g_{k}^{T} d_{k-1} \leq \tau\left\|g_{k}\right\|^{2}
$$

By using (3), we have, for all $k \geq 2$,

$$
g_{k}^{T} d_{k}=-\left\|g_{k}\right\|^{2}+\beta_{k} g_{k}^{T} d_{k-1}
$$


From the above discussion, we require that

$$
-\left\|g_{k}\right\|^{2}+\beta_{k} g_{k}^{T} d_{k-1} \leq 0 .
$$

The above inequality implies (13).

In [20], the authors proposed a variation of the FR formula:

$$
\beta_{k}^{\mathrm{VFR}}(\mu)=\frac{\mu_{1}\left\|g_{k}\right\|^{2}}{\mu_{2}\left|g_{k}^{T} d_{k-1}\right|+\mu_{3}\left\|g_{k-1}\right\|^{2}},
$$

where $\mu_{1} \in(0,+\infty), \mu_{2} \in\left[\mu_{1}+\epsilon_{1},+\infty\right), \mu_{3} \in(0,+\infty)$, and $\epsilon_{1}$ is any given positive constant. It is easy to prove that $\left\{\beta_{k}^{\mathrm{VFR}}\right\}$ is a descent sequence (with $\tau=\mu_{1} / \mu_{2}$ ) for CG methds if $g_{k}^{T} d_{k} \leq$ 0 . Formula (16) possesses the sufficient descent property and proved that there exist some nonlinear conjugate gradient formulae possessing the sufficient descent property without any line searches, where

$$
\beta_{k}^{\mathrm{WYL}}=\frac{g_{k}^{T}\left(g_{k}-\left\|g_{k}\right\| /\left\|g_{k}-1\right\|\right)}{g_{k-1}^{T} g_{k-1}} .
$$

By restricting the parameter $\sigma \leq 1 / 4$ under the SWP line search condition, the WYL method possessed the sufficient descent condition [21].

In [22], the authors designed the following variation of the PRP formula which possesses the sufficient descent property without any line searches:

$$
\begin{aligned}
& \beta_{k}^{N}(\mu) \\
& \quad= \begin{cases}\frac{\left\|g_{k}\right\|^{2}-\left|g_{k}^{T} d_{k-1}\right|}{\mu\left|g_{k}^{T} d_{k-1}\right|+\left\|g_{k-1}\right\|^{2}} & \text { if }\left\|g_{k}\right\|^{2} \geq\left|g_{k}^{T} d_{k-1}\right|, \\
0 & \text { Otherwise, }\end{cases}
\end{aligned}
$$

in which $\mu \geq 1$.

Motivated by the ideas in $[20,22]$ without any line search and sufficient descent, and taking into account the good convergence properties of [10] and the good numerical performance in [14], we propose a class new formula about $\beta_{k}$ as follows:

$$
\beta_{k}^{\mathrm{NPRP}}=\frac{\lambda \mu_{1}\left\|g_{k}\right\|^{2}+(1-\lambda) \mu_{1}\left(\left\|g_{k}\right\|^{2}-\left|g_{k}^{T} g_{k-1}\right|\right)}{\mu_{2}\left|g_{k}^{T} d_{k-1}\right|+\mu_{1}\left\|g_{k-1}\right\|^{2}},
$$

where the definitions of $\mu_{1}, \mu_{2}$ are the same as those in formula (16); $\lambda \in(0,1)$.

In order to ensure the nonnegative of the parameter $\beta_{k}$, we define

$$
\beta_{k}^{\mathrm{NPRP}^{+}}=\max \left\{0, \beta_{k}^{\mathrm{NPRP}}\right\}
$$

Thus if a negative of $\beta_{k}^{\mathrm{NPRP}}$ occurs, this strategy will restart the iteration along the steepest direction.

The following two propositions show that the $\left\{\beta_{k}^{\mathrm{NPRP}^{+}}\right\}$is a descent sequence, so that $d_{k}$ can make sufficient descent condition (9) hold.
Proposition 1. Suppose that $\beta_{k}$ is defined by (19)-(20); then one has that

$$
\beta_{k}^{N P R P^{+}} \leq \widehat{\sigma} \frac{\left\|g_{k}\right\|^{2}}{\left|g_{k}^{T} d_{k-1}\right|},
$$

where $0<\widehat{\sigma}=\mu_{1} / \mu_{2}<1$.

Proof. It is clear that inequality (21) holds when $\beta_{k}^{\mathrm{NPRP}^{+}}=0$. Now we consider the case where $\beta_{k}^{\mathrm{NPRP}^{+}}=\beta_{k}^{\mathrm{NPRP}}$. So we have

$$
\begin{aligned}
\beta_{k}^{\mathrm{NPRP}^{+}} & =\frac{\lambda \mu_{1}\left\|g_{k}\right\|^{2}+(1-\lambda) \mu_{1}\left(\left\|g_{k}\right\|^{2}-\left|g_{k}^{T} g_{k-1}\right|\right)}{\mu_{2}\left|g_{k}^{T} d_{k-1}\right|+\mu_{1}\left\|g_{k-1}\right\|^{2}} \\
& \leq \frac{\lambda \mu_{1}\left\|g_{k}^{2}\right\|+(1-\lambda) \mu_{1}\left\|g_{k}\right\|^{2}}{\mu_{2}\left|g_{k}^{T} d_{k-1}\right|} \leq \frac{\mu_{1}}{\mu_{2}} \frac{\left\|g_{k}\right\|^{2}}{\left|g_{k}^{T} d_{k-1}\right|} \\
& =\widehat{\sigma} \frac{\left\|g_{k}\right\|^{2}}{\left|g_{k}^{T} d_{k-1}\right|} .
\end{aligned}
$$

Hence $\beta_{k}^{\mathrm{NPRP}^{+}}$can make (21) hold. Furthermore $\left\{\beta_{k}^{\mathrm{NPRP}^{+}}\right\}$is a descent sequence without any line search.

Proposition 2. Suppose that $\beta_{k}$ is defined by (19)-(20); then $d_{k}$ satisfies the sufficient descent condition (9) for all $k \geq 1$, where $c=\lambda\left(1-\mu_{1} / \mu_{2}\right)$.

Proof. For any $k>1$, suppose that $g_{k-1}^{T} d_{k-1}<0$.

If $\beta_{k}^{\text {NPRP }^{+}}=0$, then $d_{k}=-g_{k}$. So we have

$$
g_{k}^{T} d_{k}=-\left\|g_{k}\right\|^{2} \leq-c\left\|g_{k}\right\|^{2}
$$

where $c=\lambda\left(1-\mu_{1} / \mu_{2}\right)$.

Otherwise, from the definition of $\beta_{k}^{\mathrm{NPRP}^{+}}$, we can obtain

$$
\begin{aligned}
& g_{k}^{T} d_{k}=g_{k}^{T}\left[-g_{k}\right. \\
& \left.+\left(\frac{\lambda \mu_{1}\left\|g_{k}\right\|^{2}+(1-\lambda) \mu_{1}\left(\left\|g_{k}\right\|^{2}-\left|g_{k}^{T} g_{k-1}\right|\right)}{\mu_{2}\left|g_{k}^{T} d_{k-1}\right|+\mu_{1}\left\|g_{k-1}\right\|^{2}}\right) d_{k-1}\right] \\
& \leq-\left\|g_{k}\right\|^{2}+\left(\frac{\lambda \mu_{1}\left\|g_{k}\right\|^{2}}{\mu_{2}\left|g_{k-1}^{T} d_{k-1}\right|}\left|g_{k}^{T} d_{k-1}\right|\right. \\
& \left.+\frac{(1-\lambda) \mu_{1}\left\|g_{k}\right\|^{2}}{\mu_{2}\left|g_{k}^{T} d_{k-1}\right|}\left|g_{k}^{T} d_{k-1}\right|\right) \leq-\left\|g_{k}\right\|^{2}+\lambda \\
& . \frac{\mu_{1}}{\mu_{2}}\left\|g_{k}\right\|^{2}+\left\|g_{k}\right\|^{2}-\lambda\left\|g_{k}\right\|^{2} \leq-\lambda\left\|g_{k}\right\|^{2}+\lambda \\
& . \frac{\mu_{1}}{\mu_{2}}\left\|g_{k}\right\|^{2} \leq-\lambda\left(1-\frac{\mu_{1}}{\mu_{2}}\right)\left\|g_{k}\right\|^{2}=-c\left\|g_{k}\right\|^{2} .
\end{aligned}
$$

For $g_{1}^{T} d_{1}=-\left\|g_{1}\right\|^{2}<0$, we can deduce that $d_{k}$ can make sufficient descent condition (9) hold for all $k \geq 1$.

By the proof of Proposition 2, we can know that the formula $\mu_{1} / \mu_{2}<1$ is necessary; otherwise, the sufficient descent condition can not be held. 


\section{Global Convergence}

In this section, we propose an algorithm related to $\beta_{k}^{\mathrm{NPRP}^{+}}$ and then we study the global convergence property of this algorithm. Firstly, we make the following two assumptions, which have been widely used in the literature to analyze the global convergence of the CG methods with the inexact line searches.

Assumption A. The level set

$$
\Omega=\left\{x \in R^{n} \mid f(x) \leq f\left(x_{1}\right)\right\}
$$

is bounded.

Assumption B. The gradient $g(x)$ is Lipschitz continuous; that is, there exists a constant $L>0$ such that, for any $x, y \in$ $\Omega$,

$$
\|g(x)-g(y)\| \leq L\|x-y\| .
$$

Now we give the algorithm.

Algorithm 3.

Step 0. Given $x_{1} \in R^{n}$, set $d_{1}=-g_{1} ; k=1$. If $g_{1}=0$, then stop. Otherwise go to Step 1.

Step 1. Find $t_{k}>0$ satisfying weak Wolfe conditions (5) and (6)

Step 2. Let $x_{k+1}=x_{k}+t_{k} d_{k}$ and $g_{k+1}=g\left(x_{k+1}\right)$. If $\left\|g_{k+1}\right\|=0$, then stop. Otherwise go to Step 3.

Step 3. Compute $\beta_{k+1}^{N P R P^{+}}$by formula (19) and (20). Then generate $d_{k+1}$ by (3).

Step 4. Set $k=: k+1$; go to Step 0 .

Since $\left\{f\left(x_{k}\right)\right\}$ is decreasing sequence, it is clear that the sequence $\left\{x_{k}\right\}$ is contained in $\Omega$, and there exists a constant $f^{*}$, such that

$$
\lim _{k \rightarrow \infty} f\left(x_{k}\right)=f^{*} .
$$

By using Assumptions $\mathrm{A}$ and $\mathrm{B}$, we can deduce that there exists $M>0$ such that

$$
\left\|g_{k}\right\| \leq M \quad \forall x \in \Omega .
$$

The following important result was obtained by Zoutendijk [1] and Wolfe [23, 24].

Lemma 4. Suppose $f(x)$ is bounded below, and $g(x)$ satisfies the Lipschitz condition. Consider any iteration method of formula (2), where $d_{k}$ satisfies $d_{k}^{T} g_{k}<0$ and $t_{k}$ is obtained by the weak Wolf line search. Then

$$
\sum_{k=1}^{\infty} \frac{\left(g_{k}^{T} d_{k}\right)^{2}}{\left\|d_{k}\right\|^{2}}<+\infty
$$

The following lemma was obtained by Dai and Yuan [25].
Lemma 5. Assume that a positive series $\left\{a_{i}\right\}$ satisfies the following inequality for all $k$ :

$$
\sum_{i=1}^{k} a_{i} \geq l k+c
$$

where $l>0$ and $c$ are constant. Then one has

$$
\sum_{i \geq 1} \frac{a_{i}^{2}}{i}=+\infty
$$

$$
\sum_{k \geq 1} \frac{a_{k}^{2}}{\sum_{i=1}^{k} a_{i}}=+\infty \text {. }
$$

Theorem 6. Suppose that Assumptions A and B hold; $\left\{x_{k}\right\}$ is a sequence generated by Algorithm 3. Then one has

$$
\liminf _{k \rightarrow \infty}\left\|g_{k}\right\|=0
$$

Proof. Equation (3) indicates that, for all $k \geq 2$,

$$
d_{k}+g_{k}=\beta_{k} d_{k-1}
$$

Squaring both sides of (33), we obtain

$$
\left\|d_{k}\right\|^{2}=-\left\|g_{k}\right\|^{2}-2 g_{k}^{T} d_{k}+\beta_{k}^{2}\left\|d_{k-1}\right\|^{2}
$$

Suppose that $\beta_{k}^{\mathrm{NPRP}^{+}}=\beta_{k}^{\mathrm{NPRP}}$ in (20). Then,

$$
\begin{gathered}
\left\|d_{k}\right\|^{2}=-\left\|g_{k}\right\|^{2}-2 g_{k}^{T} d_{k}+\left(\beta_{k}^{\mathrm{NPRP}^{+}}\right)^{2}\left\|d_{k-1}\right\|^{2} \\
=-\left\|g_{k}\right\|^{2}-2 g_{k}^{T} d_{k} \\
+\left(\frac{\lambda \mu_{1}\left\|g_{k}\right\|^{2}+(1-\lambda) \mu_{1}\left(\left\|g_{k}\right\|^{2}-\left|g_{k}^{T} g_{k-1}\right|\right)}{\mu_{2}\left|g_{k}^{T} d_{k-1}\right|+\mu_{1}\left\|g_{k-1}\right\|^{2}}\right)^{2} \\
\cdot\left\|d_{k-1}\right\|^{2} \leq-\left\|g_{k}\right\|^{2}-2 g_{k}^{T} d_{k}+\left\|g_{k}\right\|^{4} \frac{\left\|d_{k-1}\right\|^{2}}{\left\|g_{k-1}\right\|^{4}} .
\end{gathered}
$$

We have

$$
h_{k} \leq h_{k-1}-\frac{1}{\left\|g_{k}\right\|^{2}}+\frac{2 \gamma_{k}}{\left\|g_{k}\right\|^{2}} \text {, }
$$

where $h_{k}=\left\|d_{k}\right\|^{2} /\left\|g_{k}\right\|^{4}$ and $\gamma_{k}=-g_{k}^{T} d_{k} /\left\|g_{k}\right\|^{2}$.

Note that $h_{1}=1 /\left\|g_{1}\right\|^{2}$ and $\gamma_{1}=1$. It follows from (36) that

$$
h_{k} \leq-\sum_{i=1}^{k} \frac{1}{\left\|g_{i}\right\|^{2}}+2 \sum_{i=1}^{k} \frac{\left|\gamma_{i}\right|}{\left\|g_{i}\right\|^{2}} \text {. }
$$

Suppose that conclusion (32) does not hold. Then, there exists a positive scalar $\epsilon$ such that, for all $k \geq 1$,

$$
\left\|g_{k}\right\| \geq \epsilon
$$


Thus, it follows from (28) and (38) that

$$
h_{k} \leq-\frac{k}{M^{2}}+\frac{2}{\epsilon^{2}} \sum_{i=1}^{k}\left|\gamma_{i}\right| \text {. }
$$

Further, we have

$$
h_{k} \leq \frac{2}{\epsilon^{2}} \sum_{i=1}^{k}\left|\gamma_{i}\right| \text {. }
$$

On the other hand, using $h_{k} \geq 0$, relation (39) implies that

$$
\sum_{i=1}^{k}\left|\gamma_{i}\right| \geq \frac{\epsilon^{2} k}{2 M}
$$

Using Lemma 5 and (40), it follows that

$$
\sum_{k \geq 1} \frac{\left(g_{k}^{T} d_{k}\right)^{2}}{\left\|d_{k}\right\|^{2}}=\sum_{k \geq 1} \frac{\gamma_{k}^{2}}{h_{k}}=+\infty,
$$

which contradicts to Zoutendijk condition (29). This shows that (32) holds. The proof of the theorem is complete.

From the proof of the above theorem, we can conclude that any conjugate gradient method with the formula $\beta_{k}^{\mathrm{NPRP}^{+}}$ and some certain step size technique which ensures that Zoutendijk condition (29) holds is globally convergent. In particular, the formula $\beta_{k}^{\mathrm{NPRP}^{+}}$with the weak Wolfe conditions can generate a globally convergent result.

\section{Numerical Results}

All methods above are tested on 56 test problems, where the former test problems 1-48 (from arwhead to woods) in Table 1 are taken from the CUTE library in Bongartz et al. [26] and the others are taken from Moré et al. [27];

$$
\text { DYHS: } \beta_{k}=\max \left\{0, \min \left\{\beta_{k}^{\mathrm{HS}}, \beta_{k}^{\mathrm{DY}}\right\}\right\}
$$

is generated by Grippo and Lucidi [12].

All codes were written in Matlab 7.5 and run on a HP with 1.87 GB RAM and Windows XP operating system. The parameters are $\sigma=0.1, \delta=0.01, u_{1}=1, u_{2}=2$, and $\lambda=0.3$. Stop the iteration if criterion $\left\|g_{k}\right\| \leq \epsilon=10^{-6}$ is satisfied.

In Table 1, "Name" denotes the abbreviation of the test problems, " $n$ " denotes the dimension of the test problems, "Itr/NF/NG" denote the number of iteration, function evaluations, and gradient evaluations, respectively, and "Tcpu" denotes the computing time of CPU for computing a test problem (units: second).

On the other hand, to show the performance difference clearly between the hJHJ, hAN, hDY, and hHuS method, we adopt the performance profiles given by Dolan and Moré [28] to compare the performance according to Itr, NF, NG, and Tcpu, respectively. Benchmark results are generated by running a solver on a set $\mathscr{P}$ of problems and recording information of interest such as NF and Tcpu. Let $\mathcal{S}$ be the set of solvers in comparison. Assume that $\mathcal{S}$ consists of $n_{s}$

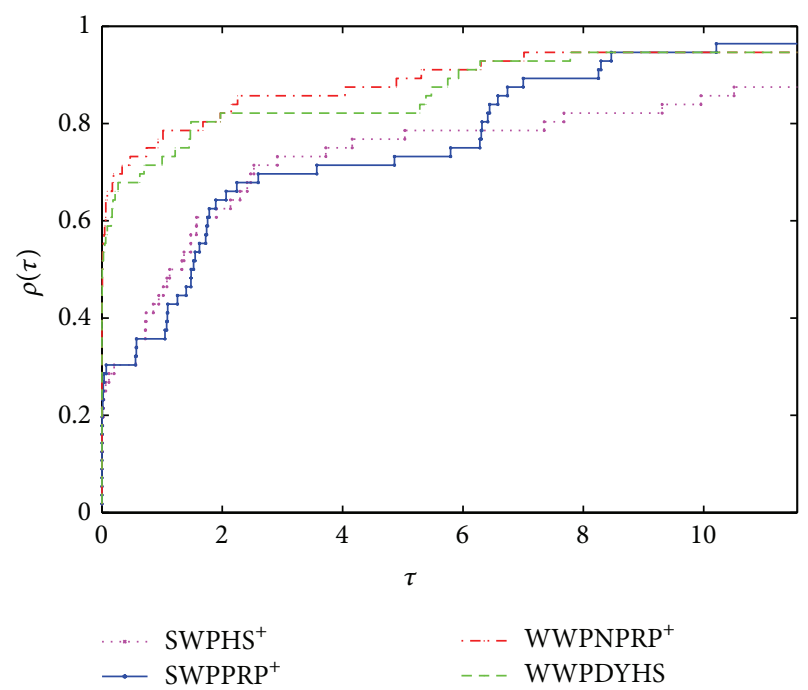

Figure 1: Performance profiles on Tpu.

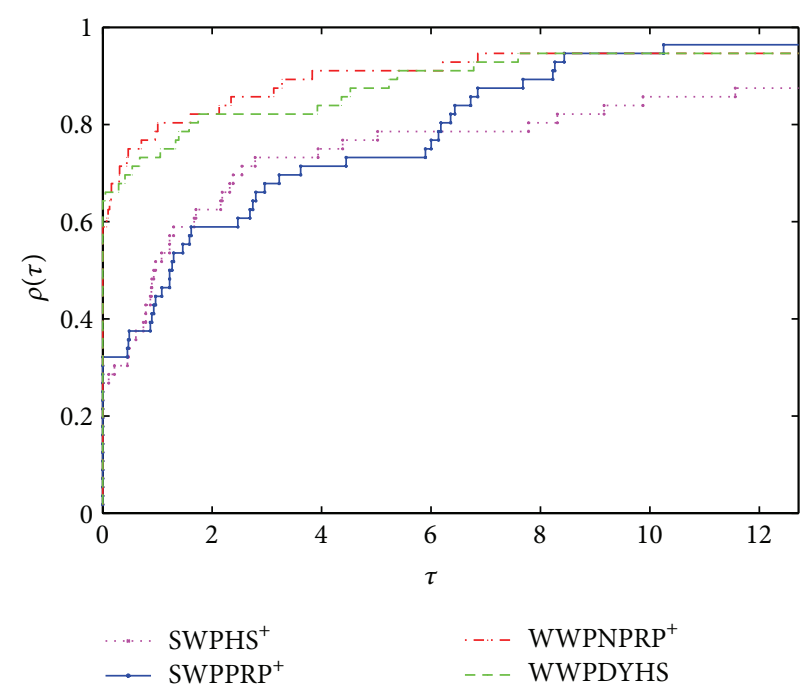

FIGURE 2: Performance profile on NF.

solvers and $\mathscr{P}$ consists of $n_{p}$ problems. For each problem $p \in \mathscr{P}$ and solver $s \in \mathcal{S}$, denote $t_{p, s}$ by the computing time (or the number of function evaluation, etc.) required to solve problem $p \in \mathscr{P}$ by solver $s \in \mathcal{S}$, and the comparison between different solvers is based on the performance ratio defined by

$$
r_{p, s}=\frac{t_{p, s}}{\min \left\{t_{p, s}: s \in \mathcal{S}\right\}} .
$$

Assume that a large enough parameter $r_{M} \geq r_{p, s}$ for all $p, s$ is chosen, and $r_{p, s}=r_{M}$ if and only if solvers $s$ do not solve problem $p$. Define

$$
\rho_{s}(\tau)=\frac{1}{n_{p}} \text { size }\left\{p \in \mathscr{P}: \log r_{p, s} \leq \tau\right\},
$$

where size $A$ means the number of elements in set $A$; then $\rho_{s}(\tau)$ is the probability for solver $s \in \mathcal{S}$ that a performance 
TABle 1: Numerical test report.

\begin{tabular}{|c|c|c|c|c|}
\hline Name/n & $\begin{array}{c}\text { SWPHS }^{+} \\
\text {Itr/NF/NG/Tcpu }\end{array}$ & $\begin{array}{c}\text { SWPPRP }^{+} \\
\text {Itr/NF/NG/Tcpu }\end{array}$ & $\begin{array}{c}\text { WWPNPRP }^{+} \\
\text {Itr/NF/NG/Tcpu }\end{array}$ & $\begin{array}{c}\text { WWPDYHS } \\
\text { Itr/NF/NG/Tcpu }\end{array}$ \\
\hline arwhead 1000 & $10 / 293 / 98 / 0.078$ & $204 / 5484 / 1606 / 1.462$ & $6 / 92 / 6 / 0.026$ & $23 / 275 / 32 / 0.073$ \\
\hline arwhead 2000 & $16 / 441 / 139 / 0.184$ & $261 / 6824 / 1825 / 2.753$ & $6 / 97 / 6 / 0.035$ & $23 / 325 / 43 / 0.135$ \\
\hline arwhead 10000 & $31 / 952 / 301 / 1.554$ & $366 / 11324 / 3139 / 17.892$ & $8 / 154 / 13 / 0.219$ & $8 / 138 / 12 / 0.206$ \\
\hline chainwoo 1000 & $11 / 329 / 91 / 0.058$ & $128 / 3745 / 1170 / 0.632$ & $26 / 305 / 43 / 0.053$ & $32 / 373 / 44 / 0.062$ \\
\hline chnrosnb 20 & $3 / 72 / 13 / 0.004$ & $3 / 72 / 13 / 0.004$ & $3 / 72 / 17 / 0.004$ & $3 / 72 / 13 / 0.004$ \\
\hline chnrosnb 50 & $6 / 149 / 37 / 0.009$ & $8 / 188 / 58 / 0.013$ & $5 / 89 / 14 / 0.005$ & $5 / 92 / 12 / 0.006$ \\
\hline cosine 1000 & $13 / 368 / 153 / 0.236$ & $3 / 113 / 38 / 0.159$ & $30 / 121 / 32 / 0.048$ & $1400 / 12416 / 1534 / 3.743$ \\
\hline cragglvy 5000 & $1 / 1 / 2 / 0.026$ & $1 / 1 / 2 / 0.026$ & $1 / 1 / 2 / 0.026$ & $1 / 1 / 2 / 0.026$ \\
\hline cragglvy 10000 & $1 / 1 / 2 / 0.027$ & $1 / 1 / 2 / 0.027$ & $1 / 1 / 2 / 0.026$ & $1 / 1 / 2 / 0.026$ \\
\hline dixmaana 3000 & $1348 / 42287 / 19153 / 185.754$ & $5 / 131 / 41 / 0.535$ & $12 / 19 / 13 / 0.128$ & $16 / 14 / 16 / 0.145$ \\
\hline dixmaanb 3000 & $13 / 47 / 23 / 0.253$ & $5 / 70 / 15 / 0.272$ & $7 / 10 / 7 / 0.066$ & $6 / 9 / 6 / 0.058$ \\
\hline dixmaanc 3000 & $14 / 49 / 31 / 0.288$ & $5 / 71 / 18 / 0.286$ & $8 / 12 / 8 / 0.077$ & $9 / 11 / 9 / 0.086$ \\
\hline dixmaand 3000 & $144 / 4122 / 1686 / 17.815$ & $5 / 72 / 21 / 0.289$ & $11 / 16 / 11 / 0.109$ & $12 / 13 / 12 / 0.116$ \\
\hline dixmaani 3000 & $\mathrm{~F} / \mathrm{F} / \mathrm{F} / \mathrm{F}$ & $4 / 98 / 33 / 0.403$ & $\mathrm{~F} / \mathrm{F} / \mathrm{F} / \mathrm{F}$ & $\mathrm{F} / \mathrm{F} / \mathrm{F} / \mathrm{F}$ \\
\hline dixmaanj 3000 & $\mathrm{~F} / \mathrm{F} / \mathrm{F} / \mathrm{F}$ & $6 / 69 / 23 / 0.305$ & $1086 / 602 / 1676 / 12.069$ & $1522 / 1420 / 2937 / 18.554$ \\
\hline dixmaanl 3000 & $13 / 355 / 134 / 1.506$ & $3 / 71 / 18 / 0.272$ & $\mathrm{~F} / \mathrm{F} / \mathrm{F} / \mathrm{F}$ & $\mathrm{F} / \mathrm{F} / \mathrm{F} / \mathrm{F}$ \\
\hline dqdrtic 1000 & $\mathrm{~F} / \mathrm{F} / \mathrm{F} / \mathrm{F}$ & $1804 / 55951 / 22587 / 6.841$ & $205 / 1470 / 205 / 0.205$ & $71 / 484 / 71 / 0.064$ \\
\hline dqdrtic 3000 & $\mathrm{~F} / \mathrm{F} / \mathrm{F} / \mathrm{F}$ & $1805 / 56040 / 22893 / 13.054$ & $182 / 1301 / 182 / 0.334$ & $96 / 648 / 96 / 0.165$ \\
\hline dqdrtic 5000 & $\mathrm{~F} / \mathrm{F} / \mathrm{F} / \mathrm{F}$ & $1805 / 56078 / 23060 / 19.535$ & $142 / 1029 / 142 / 0.390$ & $79 / 530 / 79 / 0.204$ \\
\hline dqrtic 6000 & $1 / 63 / 19 / 0.237$ & $1 / 63 / 19 / 0.238$ & $1 / 27 / 2 / 0.085$ & $1 / 27 / 2 / 0.085$ \\
\hline dqrtic 15000 & $1 / 30 / 2 / 0.234$ & $1 / 30 / 2 / 0.235$ & $1 / 30 / 2 / 0.234$ & $1 / 30 / 2 / 0.235$ \\
\hline dqrtic 20000 & $1 / 31 / 2 / 0.325$ & $1 / 31 / 2 / 0.323$ & $1 / 31 / 2 / 0.325$ & $1 / 31 / 2 / 0.324$ \\
\hline edensch 1000 & $1297 / 39852 / 18321 / 35.054$ & $1202 / 37104 / 17643 / 21.938$ & $34 / 181 / 39 / 0.171$ & $58 / 263 / 77 / 0.265$ \\
\hline engval1 3000 & $4 / 100 / 33 / 0.033$ & $608 / 18698 / 7376 / 6.380$ & $6 / 54 / 7 / 0.018$ & $17 / 112 / 33 / 0.042$ \\
\hline engvall 10000 & $4 / 100 / 28 / 0.092$ & $580 / 17809 / 6994 / 17.446$ & $7 / 58 / 10 / 0.056$ & $19 / 146 / 45 / 0.155$ \\
\hline engvall 20000 & $4 / 100 / 29 / 0.184$ & $565 / 17366 / 6871 / 33.912$ & $7 / 58 / 10 / 0.111$ & $20 / 152 / 28 / 0.304$ \\
\hline errinros 50 & $149 / 4029 / 1445 / 0.321$ & $413 / 12363 / 4708 / 0.986$ & $\mathrm{~F} / \mathrm{F} / \mathrm{F} / \mathrm{F}$ & $\mathrm{F} / \mathrm{F} / \mathrm{F} / \mathrm{F}$ \\
\hline genhumps 2000 & $32 / 566 / 245 / 0.408$ & $22 / 291 / 119 / 0.237$ & $11 / 102 / 30 / 0.071$ & $12 / 97 / 25 / 0.085$ \\
\hline genhumps 15000 & 8/178/61/1.183 & $14 / 282 / 112 / 1.767$ & $22 / 117 / 37 / 0.615$ & $13 / 187 / 56 / 1.229$ \\
\hline genhumps 20000 & $4 / 78 / 23 / 0.691$ & $5 / 102 / 36 / 0.928$ & $26 / 188 / 73 / 1.218$ & $4 / 37 / 10 / 0.275$ \\
\hline genrose 10000 & $1209 / 36119 / 12775 / 35.424$ & $2 / 63 / 14 / 0.056$ & $789 / 7297 / 792 / 7.213$ & $263 / 2365 / 265 / 2.333$ \\
\hline genrose 20000 & $1971 / 60100 / 21284 / 117.729$ & $2 / 64 / 17 / 0.119$ & $513 / 4754 / 515 / 9.346$ & $297 / 2676 / 298 / 5.283$ \\
\hline nondia 10000 & $1 / 41 / 10 / 0.028$ & $1 / 41 / 10 / 0.028$ & $1 / 21 / 2 / 0.015$ & $1 / 21 / 2 / 0.013$ \\
\hline nondia 15000 & $1 / 41 / 10 / 0.053$ & $1 / 41 / 10 / 0.044$ & $1 / 22 / 2 / 0.026$ & $1 / 22 / 2 / 0.021$ \\
\hline nondia 20000 & $1 / 42 / 9 / 0.063$ & $1 / 42 / 9 / 0.061$ & $1 / 22 / 2 / 0.029$ & $1 / 22 / 2 / 0.029$ \\
\hline nondquar 3000 & $2 / 63 / 11 / 0.208$ & $140 / 4041 / 1715 / 17.728$ & $472 / 896 / 660 / 6.186$ & $662 / 956 / 1045 / 8.098$ \\
\hline nondquar 15000 & $2 / 65 / 8 / 1.025$ & $57 / 1419 / 507 / 29.773$ & $196 / 630 / 301 / 16.855$ & $822 / 1496 / 1395 / 55.029$ \\
\hline penaltyl 100 & $17 / 439 / 318 / 0.241$ & $6 / 146 / 49 / 0.063$ & $86 / 26 / 86 / 0.072$ & $18 / 21 / 18 / 0.018$ \\
\hline penaltyl 1000 & $19 / 750 / 517 / 7.833$ & $12 / 328 / 99 / 2.655$ & $11 / 49 / 14 / 0.438$ & $24 / 65 / 29 / 0.712$ \\
\hline power1 10000 & $1 / 28 / 2 / 0.011$ & $1 / 28 / 2 / 0.012$ & $1 / 28 / 2 / 0.012$ & $1 / 28 / 2 / 0.012$ \\
\hline power1 15000 & $1 / 53 / 12 / 0.035$ & $1 / 53 / 12 / 0.036$ & $1 / 29 / 2 / 0.017$ & $1 / 29 / 2 / 0.018$ \\
\hline power1 20000 & $1 / 30 / 2 / 0.025$ & $1 / 30 / 2 / 0.025$ & $1 / 30 / 2 / 0.025$ & $1 / 30 / 2 / 0.025$ \\
\hline quartc 1000 & $1 / 54 / 17 / 0.038$ & $1 / 54 / 17 / 0.037$ & $1 / 22 / 2 / 0.013$ & $1 / 22 / 2 / 0.013$ \\
\hline quartc 6000 & $1 / 63 / 19 / 0.236$ & $1 / 63 / 19 / 0.237$ & $1 / 27 / 2 / 0.087$ & $1 / 27 / 2 / 0.085$ \\
\hline quartc 10000 & $1 / 29 / 2 / 0.152$ & $1 / 29 / 2 / 0.152$ & $1 / 29 / 2 / 0.152$ & $1 / 29 / 2 / 0.151$ \\
\hline srosenbr 5000 & $3 / 94 / 22 / 0.032$ & $190 / 5870 / 2002 / 2.140$ & $39 / 354 / 41 / 0.131$ & $7 / 81 / 8 / 0.028$ \\
\hline tridia 100 & $\mathrm{~F} / \mathrm{F} / \mathrm{F} / \mathrm{F}$ & $\mathrm{F} / \mathrm{F} / \mathrm{F} / \mathrm{F}$ & $553 / 5057 / 553 / 0.372$ & $348 / 3098 / 348 / 0.223$ \\
\hline tridia 1000 & $\mathrm{~F} / \mathrm{F} / \mathrm{F} / \mathrm{F}$ & $\mathrm{F} / \mathrm{F} / \mathrm{F} / \mathrm{F}$ & $1932 / 24283 / 1932 / 3.235$ & $1443 / 17580 / 1443 / 2.335$ \\
\hline bv 2000 & $16 / 358 / 161 / 20.822$ & $434 / 13397 / 5234 / 752.704$ & $4 / 11 / 4 / 0.636$ & $707 / 2120 / 707 / 140.003$ \\
\hline $\operatorname{lin} 010000$ & $1 / 90 / 16 / 86.643$ & $1 / 90 / 16 / 86.659$ & $1 / 65 / 2 / 58.180$ & $1 / 65 / 2 / 58.186$ \\
\hline $\operatorname{lin} 13000$ & $1 / 84 / 11 / 9.126$ & 1/84/11/9.109 & $1 / 60 / 2 / 6.184$ & $1 / 60 / 2 / 6.197$ \\
\hline $\operatorname{lin} 120000$ & $1 / 93 / 18 / 122.849$ & $1 / 93 / 18 / 122.946$ & $1 / 68 / 2 / 82.817$ & $1 / 68 / 2 / 82.707$ \\
\hline pen1 1000 & $1 / 31 / 2 / 0.780$ & $1 / 31 / 2 / 0.770$ & $1 / 31 / 2 / 0.768$ & $1 / 31 / 2 / 0.771$ \\
\hline pen1 5000 & $1 / 38 / 2 / 17.416$ & $1 / 38 / 2 / 17.415$ & $1 / 38 / 2 / 17.425$ & $1 / 38 / 2 / 17.415$ \\
\hline vardim 1000 & $1 / 68 / 2 / 0.716$ & $1 / 68 / 2 / 0.685$ & $1 / 68 / 2 / 0.689$ & $1 / 68 / 2 / 0.687$ \\
\hline vardim 5000 & $1 / 84 / 2 / 19.569$ & $1 / 84 / 2 / 20.066$ & $1 / 84 / 2 / 19.628$ & $1 / 84 / 2 / 19.720$ \\
\hline
\end{tabular}




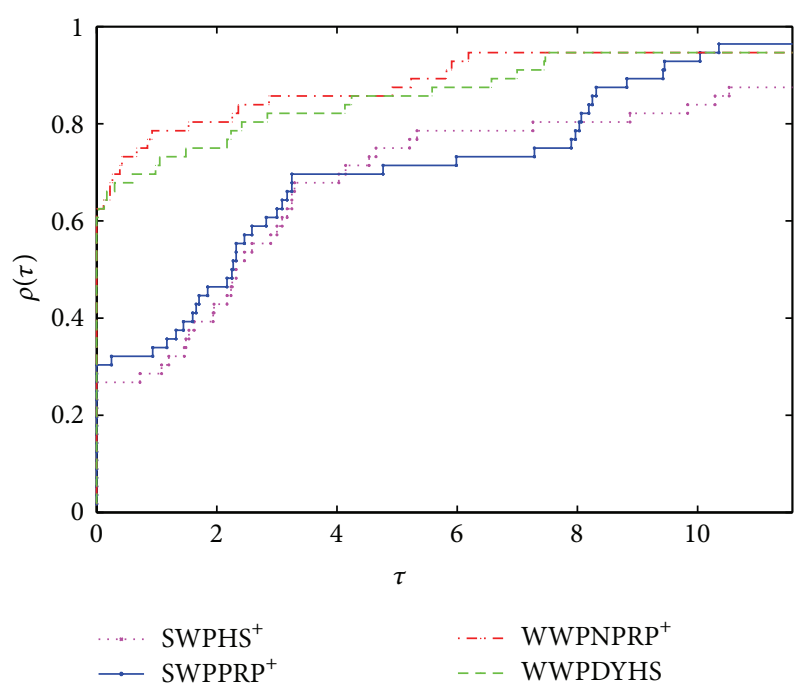

FIgURE 3: Performance profile on NG.

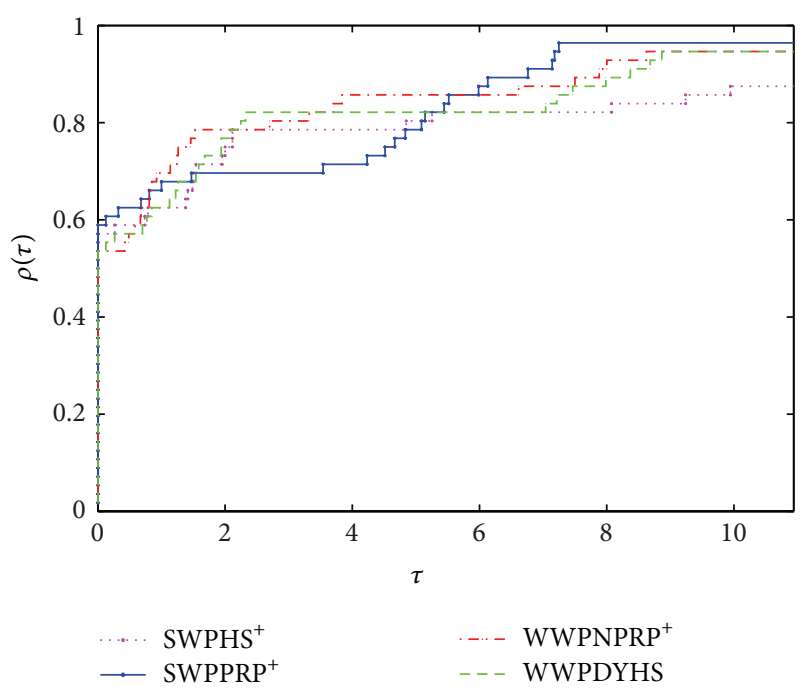

FIGURE 4: Performance profile on Itr.

ratio $r_{p, s}$ is within a factor $\tau \in R^{n}$. The $\rho_{s}$ is the (cumulative) distribution function for the performance ratio. The value of $\rho_{s}(1)$ is the probability that the solver will win over the rest of the solvers.

Based on the theory of the performance profile above, four performance figures, that is, Figures 1-4, can be generated according to Table 1. From the four figures, we can see that the NPRP is superior to the other three CGMs on the testing problems.

\section{Conclusion}

In this paper, we carefully studied the combination of the variations of the formulas $\beta_{k}^{\mathrm{FR}}$ and $\beta_{k}^{\mathrm{PRP}}$. We have found that the new formula possesses the following features: (1) $\beta_{k}^{\mathrm{NPRP}^{+}}$is a descent sequence without any line search; (2) the new method possesses the sufficient descent property and converge globally; (3) the strategy will restart the iteration automatically along the steepest descent direction if a negative value of $\beta_{k}^{\mathrm{NPRP}}$ occurs; (4) the initial numerical results are promising.

\section{Conflict of Interests}

The authors declare that there is no conflict of interests regarding the publication of this paper.

\section{Acknowledgments}

The authors are very grateful to the anonymous referees for their useful suggestions and comments, which improved the quality of this paper. This work is supported by the Natural Science Foundation of Shanxi Province (2012JQ9004), New Star Team of Xi'an University of Post and Telecommucications (XY201506), Science Foundation of Liaocheng University (318011303), and General Project of the National Social Science Foundation (15BGL014).

\section{References}

[1] G. Zoutendijk, "Nonlinear programming computational methods," in Integer and Non-Linear Programming, J. Abadie, Ed., pp. 37-86, North-Holland Publishing, Amsterdam, The Nertherlands, 1970.

[2] M. Al-Baali, "Descent property and global convergence of the fletcher-reeves method with inexact line search," IMA Journal of Numerical Analysis, vol. 5, no. 1, pp. 121-124, 1985.

[3] Y. H. Dai and Y. Yuan, "An efficient hybrid conjugate gradient method for unconstrained optimization," Annals of Operations Research, vol. 103, no. 1-4, pp. 33-47, 2001.

[4] Z. X. Wei, G. Y. Li, and L. Q. Qi, "Global convergence of the Polak-Ribière-Polyak conjugate gradient method with an Armijo-type inexact line search for nonconvex unconstrained optimization problems," Mathematics of Computation, vol. 77, no. 264, pp. 2173-2193, 2008.

[5] Y. H. Dai and Y. Yuan, "A nonlinear conjugate gradient method with a strong global convergence property," SIAM Journal on Optimization, vol. 10, no. 1, pp. 177-182, 2000.

[6] S. W. Yao, Z. X. Wei, and H. Huang, "A note about WYL's conjugate gradient method and its applications," Applied Mathematics and Computation, vol. 191, no. 2, pp. 381-388, 2007.

[7] X. Z. Jiang, G. D. Ma, and J. B. Jian, "A new global convergent conjugate gradient method with Wolfe line search," Chinese Journal of Engineering Mathematics, vol. 28, no. 6, pp. 779-786, 2011.

[8] X. Z. Jiang, L. Han, and J. B. Jian, "A globally convergent mixed conjugate gradient method with Wolfe line search," Mathematica Numerica Sinica, vol. 34, no. 1, pp. 103-112, 2012.

[9] D. Touati-Ahmed and C. Storey, "Efficient hybrid conjugate gradient techniques," Journal of Optimization Theory and Applications, vol. 64, no. 2, pp. 379-397, 1990.

[10] J. C. Gilbert and J. Nocedal, "Global convergence properties of conjugate gradient methods for optimization," SIAM Journal on Optimization, vol. 2, no. 1, pp. 21-42, 1992.

[11] Y. F. Hu and C. Storey, "Global convergence result for conjugate gradient methods," Journal of Optimization Theory and Applications, vol. 71, no. 2, pp. 399-405, 1991. 
[12] L. Grippo and S. Lucidi, "A globally convergent version of the polak-ribière conjugate gradient method," Mathematical Programming, Series B, vol. 78, no. 3, pp. 375-391, 1997.

[13] G. H. Yu, L. T. Guan, and G. Y. Li, "Global convergence of modified Polak-Ribière-Polyak conjugate gradient methods with sufficient descent property," Journal of Industrial and Management Optimization, vol. 4, no. 3, pp. 565-579, 2008.

[14] N. Andrei, "Numerical comparison of conjugate gradient algorithms for unconstrained optimization," Studies in Informatics \& Control, vol. 16, no. 4, pp. 333-352, 2007.

[15] Y. H. Dai, Analyses of conjugate gradient methods [Ph.D. thesis], Mathematics and Scientific/Engineering Computing, Chinese Academy of Sciences, 1997.

[16] G. Yu, L. Guan, and G. Li, "Global convergence of modified Polak-Ribière-Polyak conjugate gradient methods with sufficient descent property," Journal of Industrial and Management Optimization, vol. 4, no. 3, pp. 565-579, 2008.

[17] Y.-H. Dai and C.-X. Kou, "A nonlinear conjugate gradient algorithm with an optimal property and an improved wolfe line search," SIAM Journal on Optimization, vol. 23, no. 1, pp. 296320, 2013.

[18] X.-Z. Jiang and J.-B. Jian, “Two modified nonlinear conjugate gradient methods with disturbance factors for unconstrained optimization," Nonlinear Dynamics, vol. 77, no. 1-2, pp. 387-397, 2014.

[19] J. B. Jian, L. Han, and X. Z. Jiang, "A hybrid conjugate gradient method with descent property for unconstrained optimization," Applied Mathematical Modelling, vol. 39, pp. 1281-1290, 2015.

[20] Z. Wei, G. Li, and L. Qi, "New nonlinear conjugate gradient formulas for large-scale unconstrained optimization problems," Applied Mathematics and Computation, vol. 179, no. 2, pp. 407430, 2006.

[21] H. Huang, Z. Wei, and Y. Shengwei, "The proof of the sufficient descent condition of the Wei-Yao-Liu conjugate gradient method under the strong Wolfe-Powell line search," Applied Mathematics and Computation, vol. 189, no. 2, pp. 1241-1245, 2007.

[22] G. Yu, Y. Zhao, and Z. Wei, "A descent nonlinear conjugate gradient method for large-scale unconstrained optimization," Applied Mathematics and Computation, vol. 187, no. 2, pp. 636643, 2007.

[23] P. Wolfe, "Convergence conditions for ascent methods," SIAM Review, vol. 11, no. 2, pp. 226-235, 1969.

[24] P. Wolfe, "Convergence conditions for ascent methods. ii: some corrections," SIAM Review, vol. 13, no. 2, pp. 185-188, 1971.

[25] Y. Dai and Y. Yuan, Nonlinear Conjugate Methods, Science Press of Shanghai, Shanghai, China, 2000.

[26] I. Bongartz, A. R. Conn, N. Gould, and P. L. Toint, "CUTE: constrained and unconstrained testing environment," ACM Transactions on Mathematical Software, vol. 21, no. 1, pp. 123160, 1995.

[27] J. J. Moré, B. S. Garbow, and K. E. Hillstrom, "Testing unconstrained optimization software," ACM Transactions on Mathematical Software, vol. 7, no. 1, pp. 17-41, 1981.

[28] E. D. Dolan and J. J. Moré, "Benchmarking optimization software with performance profiles," Mathematical Programming, Series B, vol. 91, no. 2, pp. 201-213, 2002. 


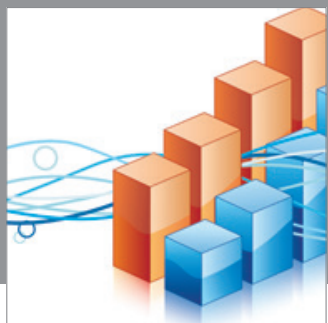

Advances in

Operations Research

mansans

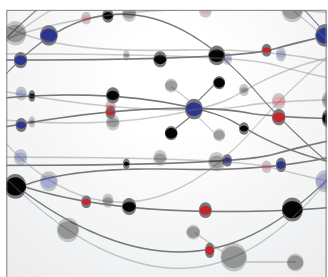

The Scientific World Journal
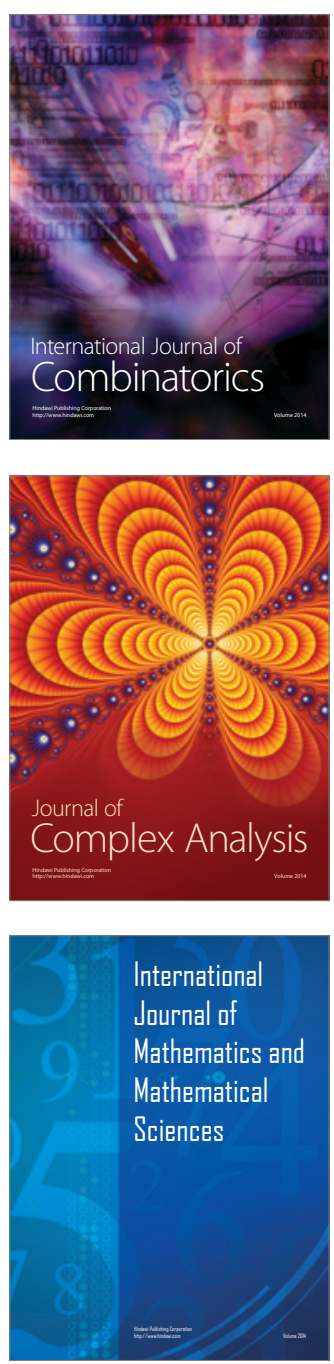
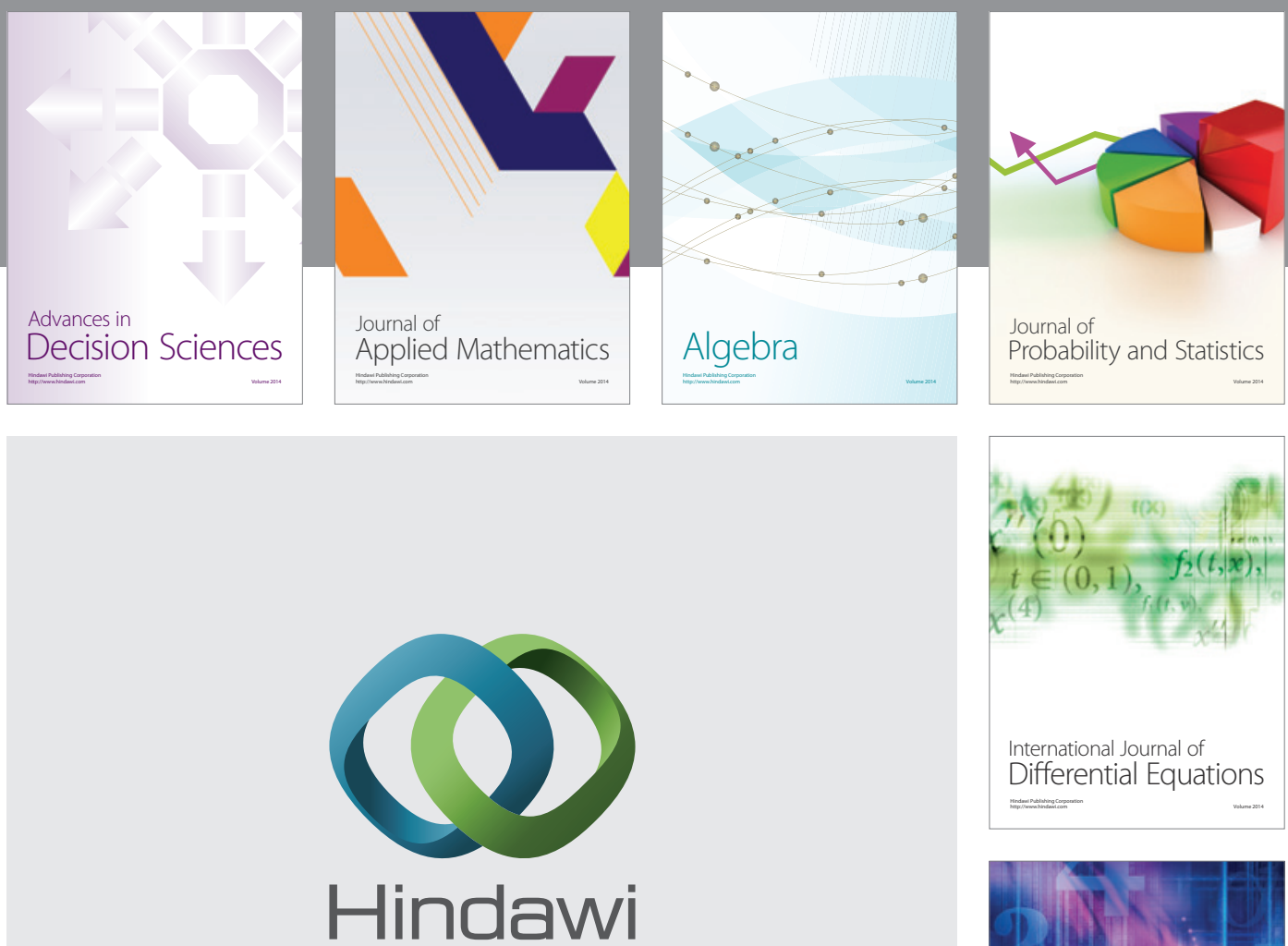

Submit your manuscripts at http://www.hindawi.com
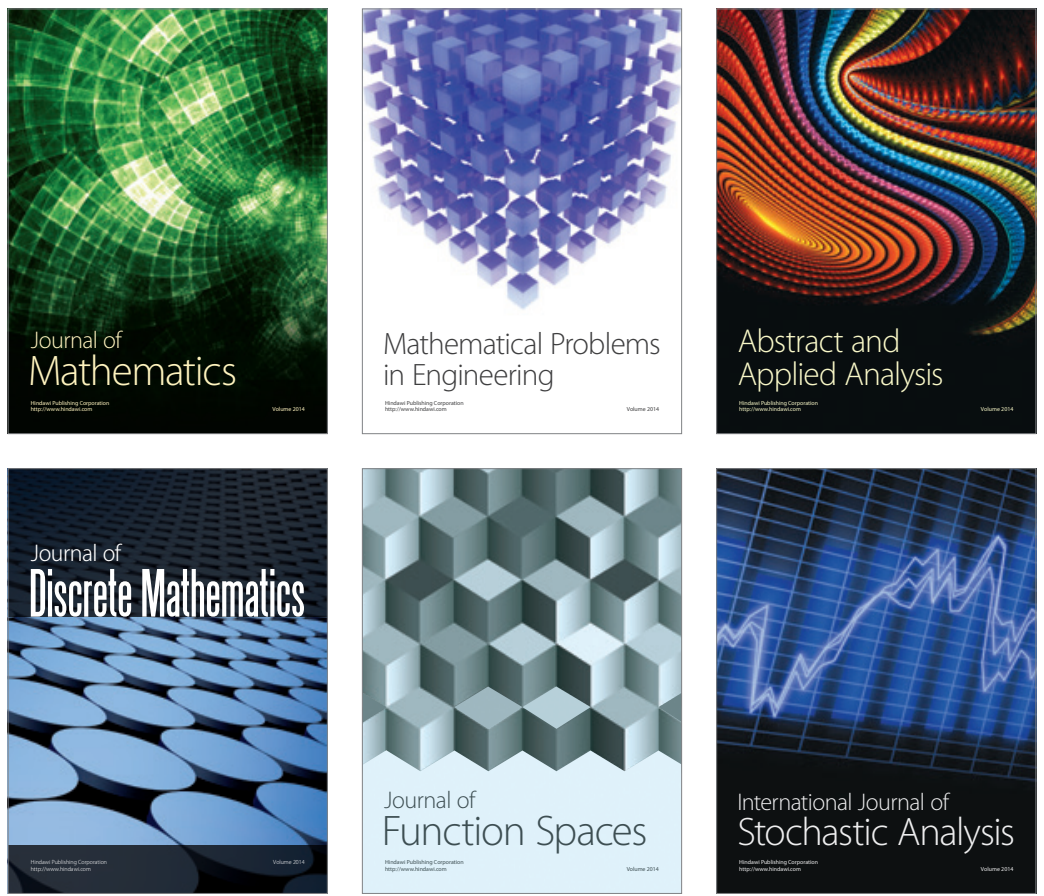

Journal of

Function Spaces

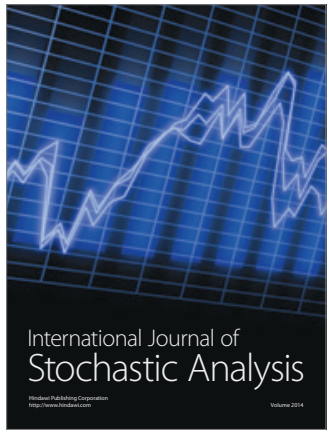

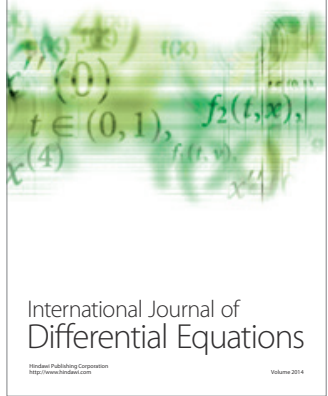
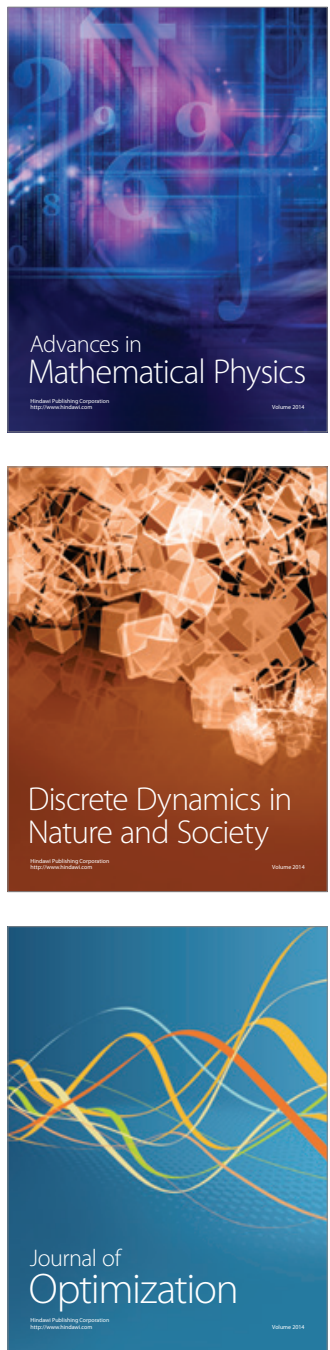